\title{
Inventory and Characterization of Mosses Diversity (Bryophyta) in Sewu Temple Yogyakarta
}

\author{
Hadi Sasongko ${ }^{1 *}$ Zuchrotus Salamah $^{2}$ Ulfatul Nurjanah ${ }^{2}$
}

\author{
${ }^{1}$ Biology Department, Science and Applied Technology Faculty, Ahmad Dahlan University, Yogyakarta, Indonesia \\ ${ }^{2}$ Biology Education Department, Faculty of Teacher Training and Education, Ahmad Dahlan University, Yogyakarta, \\ Indonesia \\ *Corresponding author. hadi.sasongko@bio.uad.ac.id
}

\begin{abstract}
The Sewu Temple area, which is located in Prambanan Yogyakarta, is a tourism site that has unique environment conditions where stone temple piles are humid and an open area. These conditions make it possible for Bryophyte to grow surrounding the area. This research aims to identify the species of Bryophyte grow in Sewu Temple Prambanan Yogyakarta and to know characteristics. The method of this research is the exploration method, which includes site investigation, identification, inventory, and descriptive analysis for both morphology and anatomy every specimen. The diversity species of mosses in the Sewu Tempel area was obtained 16 species, consisting 4 species in class Hepaticopsida, Anthocerotopsida, there are 2 species, and Bryopsida 10 species; which include : Hepaticopsida are Fossombronia other Aust., Fossombronia sp., Preissia sp., Riccia hasskarliana Steph.; Anthocerotopsida are Anthoceros two (L.) Prosk., Anthoceros punctatus L. and Bryopsidaa are Bryum coronatum Schwaegr., Bryum include: fleisch., Bryum apiculatum Schwaegr., Fissidens braunii (C.Molk) Dozy. \& Molk., Fissidens ceylonensis Dozy. \& Molk., Fissidens intromarginatulus Bartr., Hyophila involute (Hook.) Jaeg.,Barbula consanguinea (Thw. \& Mitt) Jaeg., Campylopus umbellatus (Arn.) Par., Garckea comosa (Dozy. \& Molk.) Wijk. \& Marg.

Keywords: Inventory, Bryophyte, Sewu Temple.
\end{abstract}

\section{INTRODUCTION}

Bryophyte is one of the plant species that have wide diversity, and it is considered as primitive plants with various kinds. Bryophyte can be divided into three main classes which are Hepaticae (Liverworts), Anthocerotae (Hornworts), and Musci (Mosses) [1]. It is considered as one of primitive vegetations due to its earlier appearance compared to Pteridophyte and Spermatophyte. There are more than 25,000 species of Bryophyte throughout the world [2]. Their ability to live on most of the earth's surface is because Bryophyte has a certain level of physiological endurance or dry environment, in which this species can withstand and have the endurance of a completely dry climate $[3,4]$.

Bryophyta can be found growing on any and various substrates, assist the soil stabilization through open-field colonization, play an important role in the nutrient recycling process [5]. They can withstand an extreme environment like in a hot, humid, with less supply of nutrition and water shortage. These kinds of conditions can be found in barks, woods, roof tiles, and even stones with no supply of nutrition. Candi Sewu (or Sewu Temple) is a cluster of temples located in Candi Prambanan complex, approximately 800 metres north of Roro Jonggrang Statue [6]. Many stones and statues - which is a part of the Candi Sewu temple - are found collapsed due to the increase of humidity. The humidity level on the stones triggers the spread of Bryophyte, and it also affected the stones aging process.

Some research has been done about the diversity of Bryophyte in a different habitat. The result found that there are ten kinds of Bryophyte found on location within waterfall environment [7]. Moreover, other researchproven that there are

14 species found on another temple: Candi Sukuh, Candi Borobudur, and Candi Dieng [8].

A research on the diversity of Bryophyta on Candi Sewu, according to preceding researches and publications, has never been conducted before. Especially, considering specific topics about its morphology and anatomy. This research is essential to be done in order to organise inventories of the variety of Bryophyta on the observed location. The record of species discovered in this research will give benefit even for the future, to determine the relationship between the collapse of the temple stones due to the growth of Bryophyte or to examine the ability of Bryophyte to live under a nutrition- shortage location.

\section{MATERIAL AND METHODS}

\subsection{Equipment and Material}

During the collection of the specimens, the following equipment and materials were used such as field lens, field 
note, knife, camera, pencil and pen, plastic bags, cutters, marker, and cellular phones. While during the microscopy examination, the following were used: dissecting set, dissecting microscope, binocular microscope, and glass slides, with cover slips, blades, and beaker, syringe, optilab, medicine droppers, and chloral hydrate.

\subsection{Procedure}

\subsubsection{Observation within Candi Sewu Complex}

Field observation has been done to determine the location of observation, to understand the field condition (temperature, humidity, light intensity, and soil $\mathrm{pH}$ level), and to observe the availability of Bryophyte species (liverworts, hornworts, and mosses) which has grown on the temple complex.

\subsubsection{Specimen Data Collection}

Bryophyte sample collection was completed by exploring and observing the temple area to get various types of Bryophyte. The sample was taken using spades and then was kept inside an enclosed container. Bryophyte that were discovered on the site were numerically labeled for every each of it with different morphology. Documentation was being made, as well.

\subsubsection{Observation of Morphology Characteristics}

The sample that has been collected is being cleaned gently using brushes to avoid damaging the plant structure. Morphology observation for this research consists of filloid, cauloid, and substrate type. The observed filloid organs are including colour, shape, length, edge type, and the tip filloid type. On the other hand, the parameters for cauloid organs are the length and width of cauloid.

\subsubsection{Observation of Anatomy Structure}

Plant specimen is being cleaned and placed inside a flacon tube, to soak it with chloral hydrate soluble for about two weeks to decay the chlorophyll. After that, the filloid cells of the plant are observed, including cell shape and dimension.

\subsubsection{Identification}

Identification is conducted by both morphology and anatomy observation and by matching the description of the samples to some reference about identifying Bryophyte species, which are Handbook of Malesian Mosses Vol I,
Handbook of Malesian Mosses Vol 2, Handbook of Malesian Mosses Vol 3 [9], Moss Flora of The National Botanic Garden Quezon Province, Philipines, Introduction to Bryophytes [10], and references from plant taxonomy book (Schyzophyta, Thallophyta, Bryophyte, Pteridophyta) [11]. 


\section{RESULT AND DISCUSSION}

\subsection{Result}

Table 1. Result of morphology observation

\begin{tabular}{|c|c|c|c|c|c|}
\hline No & Name of Species & Organ & Characters & Results & $\begin{array}{c}\text { Substrate } \\
\text { Type }\end{array}$ \\
\hline \multirow{5}{*}{1} & \multirow{5}{*}{$\begin{array}{l}\text { Fossombronia cristula } \\
\text { Aust }\end{array}$} & \multirow{5}{*}{ Thallus } & Colour & Green & \multirow{5}{*}{ Stones } \\
\hline & & & Shape & Frondose & \\
\hline & & & Length & $1108,296 \pm 111,972 \mu \mathrm{m}$ & \\
\hline & & & Width & $877,394 \pm 69,204 \mu \mathrm{m}$ & \\
\hline & & & Edge type & Wavy (repandus) & \\
\hline \multirow{5}{*}{2} & \multirow{5}{*}{ Fossombronia sp. } & \multirow{5}{*}{ Thallus } & Colour & Green & \multirow{5}{*}{ Stones } \\
\hline & & & Shape & Frondose & \\
\hline & & & Length & $1296,473 \pm 66,944 \mu \mathrm{m}$ & \\
\hline & & & Width & $754,374 \pm 63,520 \mu \mathrm{m}$ & \\
\hline & & & Edge type & Wavy (repandus) & \\
\hline \multirow{6}{*}{3} & \multirow{6}{*}{ Preissia sp. } & \multirow{6}{*}{ Thallus } & Colour & Green & \multirow{6}{*}{ Stones } \\
\hline & & & Shape & Frondose & \\
\hline & & & Length & $6800 \pm 1316,561 \mu \mathrm{m}$ & \\
\hline & & & Width & $3300 \pm 674,948 \mu \mathrm{m}$ & \\
\hline & & & Edge type & Wavy (repandus) & \\
\hline & & & Tip type & Split (retusus) & \\
\hline \multirow{6}{*}{4} & \multirow{6}{*}{$\begin{array}{l}\text { Riccia } \quad \text { hasskarliana } \\
\text { Steph. }\end{array}$} & \multirow{6}{*}{ Thallus } & Colour & Green & \multirow{6}{*}{ Stones } \\
\hline & & & Shape & Frondose & \\
\hline & & & Length & $8950 \pm 1802,005 \mu \mathrm{m}$ & \\
\hline & & & Width & $2600 \pm 459,468 \mu \mathrm{m}$ & \\
\hline & & & Edge type & Wavy (repandus) & \\
\hline & & & Tip type & Split (retusus) & \\
\hline \multirow{5}{*}{5} & \multirow{5}{*}{$\begin{array}{l}\text { Anthoceros leavis (L.) } \\
\text { Prosk. }\end{array}$} & \multirow{5}{*}{ Thallus } & Colour & Green & \multirow{5}{*}{ Stones } \\
\hline & & & Shape & Frondose & \\
\hline & & & Length & $7200 \pm 918,936 \mu \mathrm{m}$ & \\
\hline & & & Width & $5800 \pm 788,810 \mu \mathrm{m}$ & \\
\hline & & & Edge type & Wavy (repandus) & \\
\hline \multirow{5}{*}{6} & \multirow{5}{*}{ Anthoceros punctatus L. } & \multirow{5}{*}{ Thallus } & Colour & Green & \\
\hline & & & Shape & Frondose & \\
\hline & & & Length & $5600 \pm 966,091 \mu \mathrm{m}$ & Stones \\
\hline & & & Width & $3600 \pm 843,274 \mu \mathrm{m}$ & \\
\hline & & & Edge type & Wavy (repandus) & \\
\hline & & & Colour & Green & \\
\hline & & & Shape & Lanceolate (Eddy,1990) & \\
\hline & & & Length & $1702,524 \pm 9,120 \mu \mathrm{m}$ & \\
\hline 7 & Bryum coronatum & Filloid & Width & $471,778 \pm 51,309 \mu \mathrm{m}$ & Stones \\
\hline & & & Edge type & Flat (integer) & \\
\hline & & & Tip type & Pointed (acutus) & \\
\hline & & & Presence of midrib & Yes & \\
\hline & & & Colour & Green & \\
\hline & & & Shape & Lance (McKnight,2013) & \\
\hline & & & Length & $1627,952 \pm 16,866 \mu \mathrm{m}$ & \\
\hline 8 & Bryum erytropus fleisch. & Filloid & Width & $386,959 \pm 51,426 \mu \mathrm{m}$ & Stones \\
\hline & & & Edge type & Flat (integer) & \\
\hline & & & Tip type & Tapered (acuminatus) & \\
\hline & & & Presence of midrib & Yes & \\
\hline
\end{tabular}




\begin{tabular}{|c|c|c|c|c|c|}
\hline \multirow{7}{*}{9} & \multirow{7}{*}{$\begin{array}{l}\text { Bryum } \\
\text { Schwaegr. }\end{array}$} & \multirow{7}{*}{ Filloid } & Colour & Green & \multirow{7}{*}{ Stones } \\
\hline & & & Shape & Peat moss & \\
\hline & & & Length & $1838,61 \pm 56,228 \mu \mathrm{m}$ & \\
\hline & & & Width & $560,728 \pm 33,430 \mu \mathrm{m}$ & \\
\hline & & & Edge type & Flat (integer) & \\
\hline & & & Tip type & Tapered (acuminatus) & \\
\hline & & & Presence of midrib & Yes & \\
\hline \multirow{7}{*}{10} & \multirow{7}{*}{$\begin{array}{l}\text { Fissidens braunii } \\
\text { (C.Molk) Dozy. \&Molk. }\end{array}$} & \multirow{7}{*}{ Filloid } & Colour & Green & \multirow{7}{*}{ Stones } \\
\hline & & & Shape & Tongue & \\
\hline & & & Length & $1158,462 \pm 25,798 \mu \mathrm{m}$ & \\
\hline & & & Width & $352,117 \pm 10,404 \mu \mathrm{m}$ & \\
\hline & & & Edge type & Flat (integer) & \\
\hline & & & Tip type & Pointed (acutus) & \\
\hline & & & Presence of midrib & Yes & \\
\hline \multirow{7}{*}{11} & \multirow{7}{*}{$\begin{array}{l}\text { Fissidens ceylonensis } \\
\text { Dozy. \&Molk. }\end{array}$} & \multirow{7}{*}{ Filloid } & Colour & Green & \multirow{7}{*}{ Soils } \\
\hline & & & Shape & Lancet & \\
\hline & & & Length & $2198,892 \pm 320,931 \mu \mathrm{m}$ & \\
\hline & & & Width & $448,816 \pm 52,193 \mu \mathrm{m}$ & \\
\hline & & & Edge type & Flat (integer) & \\
\hline & & & Tip type & Pointed (acutus) & \\
\hline & & & Presence of midrib & Yes & \\
\hline \multirow{7}{*}{12} & \multirow{7}{*}{$\begin{array}{l}\text { Fissidens } \\
\text { intromarginatulus Bartr. }\end{array}$} & \multirow{7}{*}{ Filloid } & Colour & Green & \multirow{7}{*}{ Stones } \\
\hline & & & Shape & Tongue & \\
\hline & & & Length & $1918,154 \pm 72,949 \mu \mathrm{m}$ & \\
\hline & & & Width & $450,115 \pm 29,053 \mu \mathrm{m}$ & \\
\hline & & & Edge type & Flat (integer) & \\
\hline & & & Tip type & Pointed (acutus) & \\
\hline & & & Presence of midrib & Yes & \\
\hline \multirow{7}{*}{13} & \multirow{7}{*}{$\begin{array}{l}\text { Hyophila } \\
\text { (Hook.) Jaeg. }\end{array}$} & \multirow{7}{*}{ Filloid } & Colour & Pale Green & \multirow{7}{*}{ Stones } \\
\hline & & & Shape & Tongue (Alain,2009) & \\
\hline & & & Length & $2320,043 \pm 12,283 \mu \mathrm{m}$ & \\
\hline & & & Width & $728,084 \pm 19,506 \mu \mathrm{m}$ & \\
\hline & & & Edge type & Flat (integer) & \\
\hline & & & Tip type & Pointed (acutus) & \\
\hline & & & Presence of midrib & Yes & \\
\hline \multirow{7}{*}{14} & & & Colour & Green & \\
\hline & & & Shape & Lancet (McKnight,2013) & \\
\hline & & & Length & $1456,124 \pm 30,587 \mu \mathrm{m}$ & \\
\hline & Thw \& Mitt) Jaeo & Filloid & Width & $609,97 \pm 29,418 \mu \mathrm{m}$ & Stones \\
\hline & & & Edge type & Flat (integer) & \\
\hline & & & Tip type & Pointed (acutus) & \\
\hline & & & Presence of midrib & Yes & \\
\hline & & & Colour & Green & \\
\hline & & & Shape & Lancet (McKnight,2013) & \\
\hline & & & Length & $2736,29 \pm 20,222 \mu \mathrm{m}$ & \\
\hline 15 & mellatus (Arn) Par & Filloid & Width & $320,737 \pm 32,228 \mu \mathrm{m}$ & Stones \\
\hline & 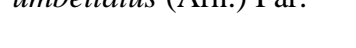 & & Edge type & Flat (integer) & \\
\hline & & & Tip type & Tapered (acuminatus) & \\
\hline & & & Presence of midrib & Yes & \\
\hline 16 & Garckea comosa & Filloid & Colour & Green & Stones \\
\hline
\end{tabular}




$\begin{array}{lll}\text { (Dozy. \&Molk.) Wijk. } & \text { Shape } & \text { Lancet (McKnight,2013) } \\ \text { \& Marg. } & \text { Length } & 2439,942 \pm 45,029 \mu \mathrm{m} \\ & \text { Width } & 500,946 \pm 16,320 \mu \mathrm{m} \\ & \text { Edge type } & \text { Flat (integer) } \\ & \text { Tip type } & \text { Pointed (acutus) } \\ & \text { Presence of midrib } & \text { Yes }\end{array}$

Table 2. Results of anatomical structure observation

\begin{tabular}{|c|c|c|c|c|c|c|c|c|}
\hline \multirow[b]{2}{*}{$\begin{array}{l}\mathbf{N} \\
\mathbf{0}\end{array}$} & \multirow[b]{2}{*}{ Name of Species } & \multirow[b]{2}{*}{ Organ } & \multirow[b]{2}{*}{ Cell shapes } & \multicolumn{4}{|c|}{ Dimensions } & \multirow[b]{2}{*}{$\begin{array}{c}\text { Length/ } \\
\text { Width } \\
(\mu \mathrm{m})\end{array}$} \\
\hline & & & & Length $(\mu \mathrm{m})$ & $\begin{array}{c}\overline{\bar{X}} \\
\text { Length } \\
(\mu \mathrm{m})\end{array}$ & $\operatorname{Width}(\mu \mathrm{m})$ & $\begin{array}{c}\bar{x} \\
\text { Widt } \\
\text { h } \\
(\mu \mathrm{m})\end{array}$ & \\
\hline \multirow{2}{*}{1} & \multirow{2}{*}{ Fossombronia cristula Aust } & Thallus & Hexagonal & $42,16 \pm 7,30$ & 42,16 & $26,75 \pm 3,22$ & 26,75 & 1,57 \\
\hline & & Midrib & - & - & - & - & - & - \\
\hline \multirow{2}{*}{2} & \multirow{2}{*}{ Fossombronia sp. } & Thallus & Hexagonal & $31,77 \pm 8,04$ & 31,77 & $21,64 \pm 4,98$ & 21,64 & 1,46 \\
\hline & & Midrib & - & - & - & - & - & - \\
\hline \multirow{2}{*}{3} & \multirow{2}{*}{ Preissia sp. } & Thallus & Hexagonal & $30,99 \pm 3,87$ & 30,99 & $22,45 \pm 2,36$ & 22,45 & 1,38 \\
\hline & & Midrib & - & - & - & - & - & - \\
\hline \multirow{2}{*}{4} & \multirow{2}{*}{ Riccia hasskarliana Steph. } & Thallus & Oval & $43,47 \pm 5,05$ & 43,47 & $22,29 \pm 7,53$ & 22,29 & 1,95 \\
\hline & & Midrib & - & - & - & - & - & - \\
\hline \multirow{2}{*}{5} & Anthoceros leavis (L.) & Thallus & Heksagonal & $36,46 \pm 4,30$ & 36,46 & $25,11 \pm 6,20$ & 25,11 & 1,45 \\
\hline & Prosk. & Midrib & - & - & - & - & - & - \\
\hline \multirow{2}{*}{6} & \multirow{2}{*}{ Anthoceros punctatus L. } & Thallus & Heksagonal & $26,27 \pm 3,15$ & 26,27 & $20,21 \pm 2,04$ & 20,21 & 1,29 \\
\hline & & Midrib & - & - & - & - & - & - \\
\hline \multirow{2}{*}{7} & coronatum & Filloid & Rectangular & $26,24 \pm 7,95$ & 26,24 & $8,58 \pm 2,25$ & 8,58 & 3,05 \\
\hline & Schwaegr. & Midrib & Rectangular & $37,83 \pm 9,44$ & 37,83 & $7,17 \pm 1,82$ & 7,17 & 5,27 \\
\hline \multirow[t]{2}{*}{8} & \multirow[t]{2}{*}{ Bryum erytropus fleisch. } & Filloid & Rectangular & $\begin{array}{c}58,22 \pm \\
12,07\end{array}$ & 58,22 & $\begin{array}{c}11,32 \pm \\
3,07\end{array}$ & 11,32 & 5,14 \\
\hline & & Midrib & Rectangular & $46,98 \pm 9,23$ & 46,98 & $5,45 \pm 1,76$ & 5,45 & 8,62 \\
\hline \multirow{2}{*}{9} & \multirow{2}{*}{$\begin{array}{l}\text { Bryum } \quad \text { apiculatum } \\
\text { Schwaegr. }\end{array}$} & Filloid & Rectangular & $26,90 \pm 7,91$ & 26,90 & $\begin{array}{c}10,82 \pm \\
1,91\end{array}$ & 10,82 & 2,48 \\
\hline & & Midrib & $\begin{array}{c}\text { Flat } \\
\text { longitudinal }\end{array}$ & $42,56 \pm 8,58$ & 42,56 & $4,62 \pm 0,78$ & 4,62 & 9,21 \\
\hline \multirow{2}{*}{10} & Fissidens braunii (C.Molk) & Filloid & Hexagonal & $8,95 \pm 1,46$ & 8,95 & $4,99 \pm 0,90$ & 4,99 & 1,79 \\
\hline & Dozy. \&Molk. & Midrib & Rectangular & $33,73 \pm 3,63$ & 33,73 & $4,02 \pm 0,64$ & 4,02 & 8,39 \\
\hline \multirow{2}{*}{11} & \multirow{2}{*}{$\begin{array}{l}\text { Fissidens ceylonensis Dozy. } \\
\text { \&Molk. }\end{array}$} & Filloid & Rectangular & $\begin{array}{c}37,43 \pm \\
10,49\end{array}$ & 37,43 & $9,54 \pm 2,03$ & 9,54 & 3,92 \\
\hline & & Midrib & Rectangular & $10,17 \pm 2,16$ & 10,17 & $2,59 \pm 0,39$ & 2,59 & 3,92 \\
\hline \multirow{2}{*}{12} & \multirow{2}{*}{$\begin{array}{l}\text { Fissidens intromarginatulus } \\
\text { Bartr. }\end{array}$} & Filloid & Isodiametrics & $6,70 \pm 1,01$ & 6,70 & $5,65 \pm 0,87$ & 5,65 & 1,18 \\
\hline & & Midrib & Rectangular & $44,41 \pm 9,03$ & 44,41 & $3,53 \pm 0,65$ & 3,53 & 12,58 \\
\hline \multirow{2}{*}{13} & Hyophila involute (Hook.) & Filloid & Square & $9,97 \pm 1,48$ & 9,97 & $7,83 \pm 1,44$ & 7,83 & 1,27 \\
\hline & Jaeg. & Midrib & Rectangular & $22,58 \pm 5,14$ & 22,58 & $4,91 \pm 0,82$ & 4,91 & 4,59 \\
\hline & Barbula consanguinea & Filloid & Rectangular & $19,80 \pm 1,93$ & 19,80 & $6,85 \pm 1,25$ & 6,85 & 2,89 \\
\hline 14 & (Thw. \& Mitt) Jaeg. & Midrib & Rectangular & $24,78 \pm 5,31$ & 24,78 & $3,35 \pm 0,46$ & 3,35 & 7,39 \\
\hline 15 & Campylopus & Filloid & Rectangular & $34,30 \pm 9,32$ & 34,30 & $\begin{array}{c}14,90 \pm \\
2,37\end{array}$ & 14,90 & 2,30 \\
\hline & & Midrib & Rectangular & $22,92 \pm 5,59$ & 22,92 & $7,83 \pm 1,11$ & 7,83 & 2,92 \\
\hline 16 & Garckea comosa (Dozy. & Filloid & Rectangular & $\begin{array}{c}43,60 \pm \\
10,62\end{array}$ & 43,60 & $5,59 \pm 1,15$ & 5,59 & 7,79 \\
\hline & \&Molk.) Wijk. \& Marg. & Midrib & Rectangular & $46,50 \pm 9,69$ & 46,50 & $6,42 \pm 0,66$ & 6,42 & 7,24 \\
\hline
\end{tabular}


Tabel 3. Identification results

\begin{tabular}{|c|c|c|c|c|c|}
\hline No & Species & Genera & Family & Order & Class \\
\hline 1 & Fossombronia cristula Aust & Foscombronia & Foscombroniaceae & Metzoeriales & \multirow{4}{*}{ Hepaticopsida } \\
\hline 2 & Fossombronia sp. & I Dasotmotomia & 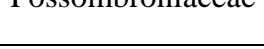 & 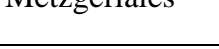 & \\
\hline 3 & Preissia sp. & Preissia & Marchantiaceae & \multirow{2}{*}{-Marchantiales } & \\
\hline 4 & Riccia hasskarliana Steph. & Riccia & Ricciaceae & & \\
\hline 5 & Anthoceros leavis (L.) Prosk. & \multirow{2}{*}{ Anthoceros } & \multirow{2}{*}{ Anthocerotaceae } & \multirow{2}{*}{ Anthocerotales } & \multirow{2}{*}{ Anthocerotopsida } \\
\hline 6 & Anthoceros punctatus L. & & & & \\
\hline 7 & Bryum coronatum Schwaegr. & \multirow{3}{*}{ Bryum } & \multirow{3}{*}{ Bryaceae } & \multirow{3}{*}{ Bryales } & \multirow{10}{*}{ Bryopsida } \\
\hline 8 & Bryum erytropus fleisch. & & & & \\
\hline 9 & Bryum apiculatum Schwaegr. & & & & \\
\hline & Fissidens braunii (C.Molk) Dozy. \&Molk. & & & & \\
\hline 11 & Fissidens ceylonensis Dozy. \&Molk. & Fissidens & Fissidentaceae & Fissidentales & \\
\hline 12 & Fissidens intromarginatulus Bartr. & & & & \\
\hline 13 & Hyophila involute (Hook.) Jaeg. & Hyophila & \multirow{2}{*}{ Pottiaceae } & \multirow{2}{*}{ Pottiales } & \\
\hline & Barbula consanguinea (Thw. \& Mitt) Jaeg. & Barbula & & & \\
\hline & Campylopus umbellatus (Arn.) Par. & Campylopus & \multirow[b]{2}{*}{ Dicranaceae } & \multirow[b]{2}{*}{ Dicranales } & \\
\hline & $\begin{array}{l}\text { Garckea comosa (Dozy. \&Molk.) Wijk. \& } \\
\text { Marg. }\end{array}$ & Garckea & & & \\
\hline
\end{tabular}




\subsection{Discussion}

The observation results show that within the Candi Sewu complex there are several Bryophyte species that belong to hornworts, liverworts, and mosses classes. This research collected 16 species of those three different groups (refer to Table 3). Most of the plants found in the location are belong to Bryopsida because the species of Bryophyte are dominated by this group. Following this group is Hepaticopsida. There are only two species of Anthocerotopsida class found on the site, mostly on the substrate area where the least species found.

The variation of Bryophyte grow on Candi Sewu can be affected by abiotic factors such as humidity, light intensity, temperature, and the $\mathrm{pH}$ level of soil or substrate. Based on the measurement result, the numbers for these mentioned parameters are obtained, which are $76 \%$ for humidity, 2475 lux of light intensity, 32oC temperature, and the average $\mathrm{pH}$ level of 6.2 for soil or substrate. Bryophyte, whether it is liverworts, hornworts, or mosses, can grow well under enough exposure of sunlight and a low substrate $\mathrm{pH}$ (alkali environment) [12, 13]. According to the result of this observation, the most common type of Bryophytes found on Candi Sewu is mosses. This division is mostly found on stones, and only one species was found in soil, which is Fissidens cevlonensis Dozy. \& Molk. Subsequently, the other species are found on a concaved side of stones and in a gap or cracks between stones. These conditions are possible due to enough level of exposure and humidity; therefore bryophytes were thriving in the area. That indicates that bryophytes are found well-grown on the environment condition of Candi Sewu.

Based on the result of descriptive analysis, it is ascertainable that there are similarities between species

\section{CONCLUSION}

The results of the study obtained 16 species of bryophytes, which belong to 3 classes, Hepaticopsida (4 species), Anthocerotopsida (2 species), and Bryopsida (10 species). Characteristics found on Bryophyte are as follows: Hepaticopsida formed of frondose thallus (like sheets), has the wavy thallus edge type and has a pleurocarp growth direction (creep). Anthocerotopsida has frondose thallus, wavy thallus edge, and has horns. In Bryopsida, it has an ascocarp type growth direction.

\section{REFERENCES}

[1] Crum. 2001. Structural Diversity of Bryophytes. University of Michigan Herbarium, Ann Arbor.

[2] Aruna, Sathisha, and Krishnappa. 2013. Bryophyte diversity in semi-evergreen forest of Chikmagalur district, Karnataka. Kuvempu University Sci. J. 2013 ISSN: 2277-9523 Volume 06, pp 01 -07 under the same division. Similarities found between the four species of liverworts are their frondose thallus shape, wavy edge thallus, and pleurocarp growth direction. The differences found for liverworts are on their length and width, which are for both morphology and anatomy structure (refer to Table 1). Preissia sp. is a liverwort which the habitat is in a humid place, sticking to soil or rocks. The talus is like a ribbon, rather thick, branched, scratched and has a middle rib that is not too prominent. The bottom side has a ventral scale and rhizoid[14].

Similarities between 2 discovered hornworts are they have frondose thallus shape, wavy thallus edge, and have hornshaped features. The difference between these types is that they have different thallus sizes and different cell dimensions. As for Anthoxeros punctatus $\mathrm{L}$ and Anthoceros leavis (L.) Prosk., it is found that they have a gravel-like thallus shape, but the thallus is thicker for Anthoceros Leavis L. Prosk.

Likenesses between ten mosses specimens found on Candi Sewu complex are that they have ascocarp (upright) growth direction, and their filloid, cauloid, and rhizoid are distinguishable. The difference between these specimens is the shape of their filloid. They have different types of filloid, for example, lancet, peat moss, and tongue. The tip filloid is different as well: they got pointed and tapered tip type. The other difference is that they got different dimensions (length and width) for both filloid features and cell features.

The cell size ratio of filloid is varied, but when observed it appears that for the Hepaticopsida and Anthocerotopsida were around one and not more than 2. While for Bryopsida (mosses), the ratio of between the length and width of the cells making up the Filloid mostly are greater than 2, and for Fissidens intromarginatulus Bartr. species it is escalated up to 12.

[3] Vitt , Crandall-Stotler, and Wood. 2017. Bryophyte: Survival in a dry world through tolerance and avoidance. In. Rajakaruna, N, R Boyd, and T Harris (eds.). Plant Ecology and Evolution in Harsh Environments. Nova Publishers.

[4] Bahaguna, Gairola, Semwal, Uniyal, and Bhatti. 2013. Bryophytes and Ecosystem. Biodiversity of Lower Plants: pp. 279-296. Editors: Rajan Kumar Gupta \& Mukesh Kumar . IK International Publishing House Pvt. Ltd.

[5] Nadhifah, Khujjah, Vitara, and Noviady. 2018. Bryophytes in Cibodas Botanical Garden: Diversity and Potential Uses. Biosaintifika 10 (2) (2018) 456-464.

[6] Perpustakaan Nasional Republik Indonesia.2004.http://candi.perpusnas.go.id/ templ es/deskripsi jawa_tengah candi_sewu. Diakses pada tanggal 11 Oktober 2017.

[7] Mundir, M.I. Elly S. Dan Agus,M.S. "Inventarisasi Lumut Terestrial Di Kawasan Wisata Air Terjun Irenggolo Kabupaten Kediri". Seminar Nasional X Pendidikan Biologi FKIP 
UNS. 12-116.

[8] Susandarini and Sujadmiko. 1997. Penggolongan lumut ditinjau dari karakter morfologi spora dengan pendekatan numerik. Biologi, 2(4) 165174.

[9] Edy, A. 1989. Handbook of Malesian Mosses Vol 1, 2,3. Natural History British Museum Publications, London.

[10] Del Rosario, Romualdo M. 1979. Moss flora of the National Botanic Garden, Quezon Province, Philippines. Agricultural Research Center, National Institute of Science and Technology, Manila.

[11] Tjitrosoepomo,G. 2009.Taksonomi Tumbuhan Schizophyta, Thallophyta, Bryophyta, Pteridophyta. Yogyakarta: UGM Press.

[12] Stuiver, Wardle, Gundale and Nilsson. 2014. The Impact of Moss Species and Biomass on the Growth of Pinus sylvestris Tree Seedlings at Different Precipitation Frequencies. Forests, 4, 5 , 1931-1951; doi:10.3390/f5081931.

[13] Jyothilakshmi, Manju, Venugopal and Chandini. 2016. Bryophyte diversity in the Sacred Groves, with special

[14] reference to Vallikkattukavu of Kozhikode district in Western Ghats. Plant Science Today 3(2): 135-141.

[15] Salamah, Hadi Sasongko and Efa Zulianti. 2018. Diversity of Bryophyte in the Selarong Cave Area, Bantul, Yogyakarta. Indonesian Journal of Biology and Education Vol. 2, No. 1, 2019, pp: 35-39 pISSN: 2654-5950, eISSN: 2654-9190.

[16] Vauzia, Syamsuardi, M. Chairul, and A. Syarif. 2016. Stomata characteristics and chlorophyll content in two plant species regenerating with sprout and seeds after at Peat Swamp Forest in Batang Alin-Indonesia. Journal of Chemical and Pharmaceutical Research. 8 (1): 356361.Budiyono, A. 2001. Pencemaran Udara: Dampak Pencemaran Udara pada Lingkungan. Berita Dkgantaia, 2 (1): 22-27. 